\title{
Clinical use of cobicistat as a pharmacoenhancer of human immunodeficiency virus therapy
}

\author{
This article was published in the following Dove Press journal: \\ HIVIAIDS - Research and Palliative Care \\ 22 December 2015 \\ Number of times this article has been viewed
}

\section{Nils von Hentig}

HIV Center, Medical Department II, Hospital of the JW Goethe-University, Frankfurt, BAG Darab-Kaboly/von Hentig, General Medicine and HIV Care, Frankfurt am Main, Germany
Correspondence: Nils von Hentig Department of Internal Medicine II, HIV Center, Johann Wolfgang Goethe University, Theodor-Stern-Kai 7, 60590 Frankfurt am Main, Germany, BAG Darab-Kaboly/von Hentig, Ziegelhüttenweg I-3, 60598 Frankfurt am Main, Germany $\mathrm{Tel}+49696|403|$

Fax +496960325884

Email hentig@em.uni-frankfurt.de

\begin{abstract}
The pharmacoenhancement of plasma concentrations of protease inhibitors by coadministration of so-called boosters has been an integral part of antiretroviral therapy for human immunodeficiency virus (HIV) for 1.5 decades. Nearly all HIV protease inhibitors are combined with low-dose ritonavir or cobicistat, which are able to effectively inhibit the cytochrome-mediated metabolism of HIV protease inhibitors in the liver and thus enhance the plasma concentration and prolong the dosing interval of the antiretrovirally active combination partners. Therapies created in this way are clinically effective regimens, being convenient for patients and showing a high genetic barrier to viral resistance. In addition to ritonavir, which has been in use since 1996, cobicistat, a new pharmacoenhancer, has been approved and is widely used now. The outstanding property of cobicistat is its cytochrome P450 3A-selective inhibition of hepatic metabolism of antiretroviral drugs, in contrast with ritonavir, which not only inhibits but also induces a number of cytochrome P450 enzymes, UDP-glucuronosyltransferase, P-glycoprotein, and other cellular transporters. This article reviews the current literature, and compares the pharmacokinetics, pharmacodynamics, and safety of both pharmacoenhancers and discusses the clinical utility of cobicistat in up-to-date and future HIV therapy.
\end{abstract}

Keywords: human immunodeficiency virus, fixed-dose combinations, pharmacoenhancers, drug safety

\section{Introduction}

The introduction of combination antiretroviral therapy (cART) in 1996 greatly decreased the overall morbidity and mortality of patients with human immunodeficiency virus (HIV) worldwide. ${ }^{1,2}$ As the eradication of HIV after infection and a cure is still not possible, long-term viral efficacy, safety, tolerability, and convenience are the major goals of probable lifelong HIV treatment. The disadvantages of long-term treatment related to protease inhibitor (PI)-based cART are multiple, in particular metabolic disorders ${ }^{3}$ and cardiovascular disease, ${ }^{4-6}$ and have changed the view of clinicians on such regimens. Given that cART has to be taken by patients over decades, drugs are not only chosen because of their antiretroviral potency but also assessed for their possible long-term side effects, pill burden, and potential for interactions with other commonly taken drugs. In particular, the growing number of patients older than 60 years of age, family planning or pregnant women or, most vulnerable, children are in need of effective and safe long-lasting cART. ${ }^{7,8}$ Further, treatment of hepatitis coinfection with new PIs for hepatitis $\mathrm{C}$ virus, management of metabolic syndrome, and the growing number of salvage patients has drawn attention to the pharmacokinetic and pharmacodynamic interactions within such regimens. ${ }^{9-13}$ One key question is whether

submit your manuscript $\mid$ www.dovepress.con 
the principle of boosting or the booster itself causes some of the long-term side effects.

\section{Current HIV therapy regimen}

Almost all PIs and non-nucleoside reverse-transcriptase inhibitors (NNRTIs) used to treat HIV, along with maraviroc, are metabolized by intestinal and hepatic cytochrome oxidases, in particular P450 (CYP)3A. Administered orally as single drugs, PIs in particular have low systemic exposure and short half-lives. In 1996, it was shown that ritonavir, an HIV-1 PI with marked antiviral activity, was able to inhibit CYP3A isoenzymes ${ }^{14}$ and thus enhance the plasma exposure of combination partners such as indinavir, saquinavir, or lopinavir, ie, the first-generation HIV PIs. Today, ritonavir is administered at low subtherapeutic doses of $100 \mathrm{mg}$ as a cART-boosting agent together with a second-generation PI, ie, atazanavir or darunavir. ${ }^{15}$ Although widely used, coadministration with ritonavir causes a number of problems; it is able to inhibit or induce other CYP subfamilies, eg, CYP2D6, CYP2C9, CYP2C19, and CYP1A2, ${ }^{14}$ urunosyl-glucuronyltransferase (UGT), and transmembrane drug transporters, eg, OCT and ABCB1 (P-glycoprotein), so is responsible for a large number of drug-drug interactions. Even the low dose of $100 \mathrm{mg}$ once daily can cause unfavorable, in particular gastrointestinal, side effects over a long time.

Hence, other HIV-1 PI-boosting drugs have been searched for, and a new entity, cobicistat, was finally approved in 2012. The unique pharmacological property of cobicistat is its selective inhibition of CYP3A, the renal transmembrane transporter (MATE-1) and intestinal ABCB1 at low concentrations $(0.034 \mu \mathrm{mol}) .{ }^{16,17}$ Cobicistat also has unwanted side effects, albeit to a lesser extent than ritonavir, and its potential to decrease creatinine clearance in particular has been discussed extensively in the recent past. Therefore, this review of cobicistat as a pharmacoenhancer pays special attention to its mechanisms of action, safety profile, and clinical utility in the long-term perspective.

\section{Pharmacological properties of HIV pharmacoenhancers}

\section{Mechanisms of action}

Ritonavir was designed in the early 1990s as an HIV PI in order to inhibit the dissection of viral protein precursors of HIV-1-infected cells by viral proteases, thereby stopping the production of viral particles and subsequent infection of uninfected cells. ${ }^{18}$ Initially, $600 \mathrm{mg}$ ritonavir was administered orally twice daily together with nucleoside reverse transcriptase inhibitors (mainly epivir, zidovudine, or didanosine). ${ }^{19,20}$ In combination with other early firstgeneration PIs (saquinavir, ${ }^{21,22}$ indinavir ${ }^{23,24}$ ) in patients failing therapy with ritonavir alone, plasma PI concentrations of the coadministered drugs were much higher than previously expected. Patients could decrease the dosing frequency and the dose of both HIV PIs and, even more important, such combinations could overcome previously archived viral resistance against single PIs. ${ }^{21}$ Today, ritonavir is formulated as a pharmacoenhancer at a low dose of $100 \mathrm{mg}$.

Cobicistat on the other hand is antiretrovirally inactive and selectively inhibits CYP3A isoenzymes (but not other cytochromes or UGT), and also a number of transmembrane drug transporters at different blood-target tissue barriers. Cobicistat is coformulated into fixed-dose combinations ${ }^{12}$ in order to reduce pill burden and improve adherence with once-daily HIV regimens, ${ }^{25-29}$ but is also available as a single agent $\left(\right.$ Tybost $\left.^{\circledR}\right)$.

Several Phase I studies have shown the effective pharmacoenhancing potential of cobicistat. In the first studies in healthy volunteers, the pharmacokinetic parameters of elvitegravir following administration of a fixed-dose combination with elvitegravir, emtricitabine, tenofovir disoproxil fumarate (DF), and cobicistat were similar to those of ritonavir-boosted elvitegravir. ${ }^{30}$ In addition, plasma concentrations of the HIV PI atazanavir were comparable between regimens containing atazanavir/ritonavir (300/100 mg) and those containing atazanavir/cobicistat (300/150 mg). ${ }^{31}$ Further, the comparable efficacy and safety of cobicistat versus ritonavir when taken together with atazanavir/emtricitabine/tenofovir DF (ATV/ FTC/TDF 300/200/245 mg) were confirmed in cART-naïve, HIV-1-infected patients. ${ }^{17,32}$

The pharmacokinetic parameters of darunavir following coadministration of once-daily darunavir $800 \mathrm{mg}$ with cobicistat $150 \mathrm{mg}$, either as single agents ${ }^{33}$ or as two candidate fixed-dose combinations (G003 and G004), ${ }^{34}$ were similar to those of darunavir/ritonavir (800/100 mg) administered once daily, except for lower plasma darunavir concentrations after 24 hours with darunavir/cobicistat. ${ }^{23,24}$

\section{Drug-drug interactions of Pls}

The potential for pharmacokinetic interactions is always a major concern of cART for clinicians. Not only PIs, but also non-nucleoside reverse transcriptase inhibitors (NNRTIs, efavirenz, etravirine, rilpivirine, and nevirapine), the CCR5 inhibitor maraviroc, and the new integrase inhibitor elvitegravir, are metabolized in part by the same hepatic CYP oxidases and are consequently vulnerable to changes in CYP metabolic activity. Boosting of HIV PIs with ritonavir 100 
$\mathrm{mg}$ or $200 \mathrm{mg}$ has been described extensively in the past, ${ }^{35-39}$ but ritonavir also affects CYP2D6, CYP2C9, CYP2C19, and CYP1A2, and these effects can be quite variable; starting with marginal effects in combination with lopinavir, ${ }^{40,41}$ it can result in considerable effects if coadministered with atazanavir/saquinavir. ${ }^{42}$ In the contrary, the lopinavir/ritonavir combination itself is vulnerable to inducers of CYP3A metabolism, in that nevirapine and efavirenz, for example, both substantially decrease the plasma exposure of lopinavir and ritonavir when coadministered..$^{43-45}$

In contrast, the new booster cobicistat was selected from a number of progenitors because of its selective inhibition of CYP3A. It inhibits human hepatic and intestinal CYP3A activity at a very low inhibitory concentration (IC), and the $\mathrm{K}_{1}$ and $k_{\text {inact }}$ are comparable with those of ritonavir. Like ritonavir, cobicistat is a substrate for hepatic CYP3A metabolism. However, unlike ritonavir, cobicistat is only a weak inhibitor of CYP2D6 (at an $\mathrm{IC}_{50}$ of $9.2 \mu \mathrm{mol} / \mathrm{L}$ ) and does not affect CYP1A2, CYP2C9, or CYP2C19 $\left(\mathrm{IC}_{50}>25 \mu \mathrm{mol} / \mathrm{L}\right)$.

In contrast with ritonavir, which induces early phase I cytochrome expression after therapy is initiated, as well as phase II UGT activity, inducing its own metabolism and that of coadministered drugs for up to 2 weeks until a steady state is reached, cobicistat has no such influence in vivo.

\section{Cobicistat versus ritonavir}

Both ritonavir and cobicistat given at low doses (100 mg and $50 \mathrm{mg}$, respectively) were compared during the pivotal trials for cobicistat. Their efficacy was shown to be comparable; however, the safety aspects showed differences between the two drugs. Lipodystrophy or the potential induction of insulin resistance when ritonavir is combined with other prevalent PIs during long-term cART has not been seen with cobicistat so far, albeit the period of experience with cobicistat as a
cART combination partner is much shorter than that with ritonavir, so its potential to induce lipodystrophy may not be fully appraised as yet. On the other hand, cobicistat significantly increased serum creatinine concentrations during the early phase of treatment and raised concerns about its potential renal toxicity. The two drugs share a similar potential for gastrointestinal toxicity, although a lower risk for diarrhea has been found for cobicistat. These issues, along with other side effects presented, are discussed in the following sections.

\section{Pharmacokinetics, safety, and pharmacodynamics of cobicistat Phase I pharmacokinetics (GS-2I6-0I0I)}

The pharmacokinetics of cobicistat were investigated for the first time in 84 healthy male volunteers who received cobicistat 50, 100, 200, 300, and $400 \mathrm{mg}$ in a sequential study with escalating single doses of cobicistat and in 72 volunteers who took repeated doses of 50, 100, 200, and $300 \mathrm{mg}$ at steady state. Steady-state pharmacokinetics showed both dose-dependent and time-dependent changes in the pharmacokinetic parameters of cobicistat, leading to an up to 47-fold increase in cobicistat exposure and a $95 \%$ decrease in clearance after multiple dosing with 50-300 mg $(P<0.001$, analysis of variance, see Table 1$)$.

Three subjects in each group received ritonavir $100 \mathrm{mg}$ or placebo in order to evaluate and compare the inhibitory potential of either ritonavir or cobicistat on concomitantly administered midazolam, which is known to be a selective CYP3A substrate. Clearance of midazolam was reduced by $89 \%$ when coadministered with cobicistat, which was comparable with that seen for $100 \mathrm{mg}$ of ritonavir (see Table 2). Twelve subjects discontinued the study treatments, but only one stopped early due to a drug-related adverse event.

Table I Pharmacokinetic parameters of cobicistat at steady state (GS-216-0I0I study)

\begin{tabular}{|c|c|c|c|c|}
\hline Parameters & $50 \mathrm{mg}$ & $100 \mathrm{mg}$ & $200 \mathrm{mg}$ & $300 \mathrm{mg}$ \\
\hline Multiple dose & $n=12$ & $n=I I$ & $n=12$ & $n=12$ \\
\hline $\mathrm{C}_{\max }(\mathrm{ng} / \mathrm{mL})$ & $170(70.1)$ & $563(30.7)$ & $\mathrm{I}, 850(28.0)$ & $3,840(16.7)$ \\
\hline AUC $\tau$ (ng*hour/mL) & $827(81.6)$ & $3,440(34.3)$ & $16,100(34.3)$ & $39,100(27.6)$ \\
\hline $\mathrm{T}_{\max }$ (hours) & $4.50(3.50 ; 4.50)$ & $4.50(4.50 ; 4.53)$ & $4.50(4.50 ; 4.50)$ & $4.00(3.25 ; 4.50)$ \\
\hline $\mathrm{t}_{1 / 2}$ (hours) & $2.19(1.34 ; 2.48)$ & $3.12(2.55 ; 3.36)$ & $5.20(4.12 ; 6.10)$ & 8.07 (5.89; 9.01$)$ \\
\hline $\mathrm{CL}_{\mathrm{ss}} / \mathrm{F}$ (L/hour) & $154(106.9)$ & $33.2(43.5)$ & $14.0(38.4)$ & $8.28(29.9)$ \\
\hline $\mathrm{V}_{\mathrm{ss}}^{\mathrm{ss}} / \mathrm{F}(\mathrm{L})$ & $316(69.0)$ & $152(65.9)$ & $100(15.9)$ & $85.3(15.0)$ \\
\hline Accumulation index & $3.12(2.37 ; 4.11)$ & $2.10(1.74 ; 2.50)$ & $1.97(1.75 ; 2.23)$ & $1.88(1.67 ; 2.12)$ \\
\hline
\end{tabular}

Note: All parameters are shown as the mean $(\% \mathrm{Cl})$, except for $\mathrm{T}_{\max }$ and $\mathrm{t}_{1 / 2}$ (median, first and third quartile). ${ }^{30}$

Abbreviations: $\mathrm{C}_{\max }$, maximum observed plasma concentration during the dosing interval; $\mathrm{Cl}$, confidence intervals; $\mathrm{AUC} \tau$, area under the time concentration curve over the dosing interval; $\mathrm{C} \tau$, plasma concentration at the end of the dosing interval; $\mathrm{T}_{\max ,}$ time to reach maximum plasma concentration during the dosing interval; $\mathrm{t}_{1 / 2}$, half-life; $\mathrm{CL}_{\mathrm{ss}} / \mathrm{F}$, clearance at steady state; $\mathrm{V}_{\mathrm{ss}} / \mathrm{F}$, volume of distribution at steady state. 
Table 2 Clearance of the CYP3A substrate midazolam, either combined with cobicistat or alone, reported as the GMR and $90 \% \mathrm{Cl}^{30,72}$

\begin{tabular}{lll}
\hline Drug and dose & GMR (90\% CI) & Mean CL reduction (\%) \\
\hline Cobicistat $50 \mathrm{mg}$ & II.5 (I0.I; I3.2) & 88.5 \\
Cobicistat $100 \mathrm{mg}$ & $7.3(6.3 ; 8.4)$ & 92.7 \\
Cobicistat $200 \mathrm{mg}$ & $5.2(4.5 ; 6.0)$ & 95.8 \\
Ritonavir $100 \mathrm{mg}$ & $4.5(3.7 ; 5.4)$ & 97.5 \\
\hline
\end{tabular}

Abbreviations: $\mathrm{Cl}$, confidence interval; $\mathrm{CL}$, clearance; CYP, cytochrome P450; GMR, geometric mean ratio.

\section{Phase I safety (GS-2I6-0I2I)}

Thirty-six HIV-negative adult volunteers took cobicistat $150 \mathrm{mg}$ plus ritonavir placebo $(\mathrm{n}=12)$, ritonavir $100 \mathrm{mg}$ plus cobicistat placebo $(\mathrm{n}=12)$, or ritonavir placebo plus cobicistat placebo $(n=12)$ for 7 days to investigate and quantify the effect of each drug on kidney function. In addition, 18 volunteers with moderately impaired renal function (estimated glomerular filtration rate [GFR] $50-79 \mathrm{~mL} / \mathrm{min}$ ) took cobicistat $150 \mathrm{mg}$ for 7 days without placebo. A 1,500 $\mathrm{mg}$ bolus of iohexol, a substance that is almost $100 \%$ filtered at the glomerulus and unlike creatinine is not secreted in the kidney tubules, thus representing the reference substance for the real change in GFR in humans, was injected intravenously on days 0,7 , and 14 (follow-up) and the iohexol plasma concentration was measured. The clearances of ritonavir and cobicistat were calculated at day 7, and the GFR was calculated using the Cockcroft-Gault formula $\left(\mathrm{GFR}_{\mathrm{CG}}\right){ }^{46}$ Subsequently, it was shown that the estimated GFR changed significantly $(-9.90 \mathrm{~mL} / \mathrm{min}, P<0.05)$, but not the apparent $\mathrm{GFR}_{\mathrm{CG}}(-2.70 \mathrm{~mL} / \mathrm{min})$. The group with reduced renal function also showed a statistically significant reduction in estimated GFR $(-11.9 \mathrm{~mL} / \mathrm{min} ; P<0.001)$ and apparent $\mathrm{GFR}_{\mathrm{CG}}$ $(-3.60 \mathrm{~mL} / \mathrm{min})$, whereas the administration of ritonavir did not cause any changes in the GFR in either group. ${ }^{46}$

\section{Phase II pharmacodynamics and safety (GS-216-0 I05)}

In this randomized controlled clinical study, the safety and efficacy of cobicistat-boosted ( $\mathrm{n}=50$ ) versus ritonavir-boosted $(\mathrm{n}=29)$ atazanavir $(300 \mathrm{mg}$ ) was investigated in HIV-1infected adults through 48 weeks on therapy. The rates of grade 1-4 adverse events seen with cobicistat (36\%) and ritonavir (48\%) were comparable. Only two patients discontinued the cobicistat-based therapy regimen due to adverse events (vomiting, rash) and one patient stopped taking ritonavir because of ocular icterus, most likely related to taking ritonavir-boosted atazanavir. Approximately $5 \%$ of patients in each study group developed grade 2-4 laboratory abnormalities, including increased total bilirubin (likely caused by inhibition of bilirubin-metabolizing UGT1A1 by atazanavir), serum amylase, total cholesterol, and creatine kinase concentrations, decreased serum phosphate levels, and hematuria. Small to moderate changes in total, high-density lipoprotein cholesterol, low-density lipoprotein cholesterol, and triglycerides were comparable between the two study groups. Further, mean estimated GFR decreased by $13.3 \mathrm{~mL} / \mathrm{min}$ in patients taking cobicistat and by $13.8 \mathrm{~mL} / \mathrm{min}$ in those receiving ritonavir as part of their cART. ${ }^{17,47}$

\section{Pharmacodynamics and safety (GS-236-0104)}

GS 236-0104 was a randomized, controlled, Phase II clinical trial evaluating the safety and efficacy of elvitegravir $150 \mathrm{mg} /$ cobicistat $150 \mathrm{mg} /$ emtricitabine $200 \mathrm{mg} /$ tenofovir-DF $245 \mathrm{mg}$ in a fixeddose tablet ("QUAD", marketed as Stribild ${ }^{\circledR}, \mathrm{n}=48$ ) versus efavirenz $600 \mathrm{mg}$ /emtricitabine $200 \mathrm{mg}$ /tenofovir-DF $245 \mathrm{mg}$ as a fixed-dose combination (Atripla ${ }^{\circledR}, n=23$ ).

At baseline, patients in both groups were of comparable mean age (36 versus 35 years), mean viral load (HIV-RNA polymerase chain reaction $4.59 \log _{10}$ versus $4.58 \log _{10}$ copies $/ \mathrm{mL}$ ), and median CD4 cell count (354 versus 436 cells $/ \mathrm{mm}^{3}$ ).

The incidence of drug-related adverse events through 48 weeks on therapy was also comparable in both groups ( $46 \%$ versus 57\%). Two patients in either arm experienced grade 3-4 adverse events, without discontinuing the study. However, patients taking the QUAD tablet experienced significantly fewer central nervous system (CNS) symptoms compared with patients taking efavirenz/emtricitabine/tenofovir-DF ( $17 \%$ versus $43 \%, P=0.02$ ) and fewer psychiatric symptoms (10\% versus $26 \%)$. One patient on efavirenz-containing therapy stopped treatment due to suicidal ideation. ${ }^{16}$ In this trial, no grade 3-4 laboratory adverse events were reported, and cholesterol/triglyceride increases did not exceed grade 1-2. ${ }^{16}$

Again, the estimated $\mathrm{GFR}_{\mathrm{CG}}$ decreased, being $19.7 \mathrm{~mL} / \mathrm{min}$ in patients taking cobicistat versus $5.5 \mathrm{~mL} / \mathrm{min}$ in patients taking the unboosted therapy regimen. However, none of the study patients stopped early due to changes in the estimated GFR or related serum creatinine. ${ }^{16}$

\section{Phase I kidney safety study}

Subsequently, a Phase I trial was conducted to evaluate the effects of cobicistat on GFR. A group of healthy volunteers took cobicistat or placebo ( $\mathrm{n}=12$ in each group) for 1 week with a washout period of 7 days between the study medications. Again, estimated GFR decreased when cobicistat was taken, but returned to baseline after intake of the study drug was 
stopped. A parallel group of volunteers received iohexol, the reference compound for detection of changes in GFR. However, the iohexol clearance was not affected at all. ${ }^{46}$

The investigators concluded that there was a cobicistatrelated decrease in renal SLC22A2 or SLC47A1 transmembrane transporter activity, which is part of the tubular excretion pathway for creatinine. Logically, this effects an increase of serum creatinine concentrations, distorting the estimation of GFR by the Cockcroft-Gault formula, but not the real GFR, thus showing a nonexistent effect on glomerular filtration caused by cobicistat. ${ }^{30}$

\section{Phase III safety and efficacy GS- 1020}

GS-1020, a controlled Phase III clinical study, evaluated the safety and efficacy of elvitegravir $150 \mathrm{mg} /$ cobicistat $150 \mathrm{mg} /$ emtricitabine $200 \mathrm{mg} /$ tenofovir-DF $245 \mathrm{mg}$ as a fixed-dose combination (Stribild, $n=348$ ) versus efavirenz $600 \mathrm{mg} / \mathrm{em}$ tricitabine $200 \mathrm{mg} /$ tenofovir-DF $245 \mathrm{mg}$ (fixeddose combination, Atripla, $n=352$ ). At baseline, patients in both study groups were of mean age 38 years, with a similar viral load (HIV-RNA polymerase chain reaction $4.75 \log _{10}$ versus $4.78 \log 10$ copies $/ \mathrm{mL}$ ) and comparable median absolute CD4 count (391 versus 382 cells $/ \mathrm{mm}^{3}$ ). The proportions of male ( $88 \%$ versus $90 \%$ ) and asymptomatic HIV-infected patients ( $83 \%$ versus $84 \%$ ) were also comparable at baseline between the groups, and representative of the typical treatment-naïve patient starting first-line therapy according to the majority of the international guidelines for the treatment of HIV-1-infected adults. However, the proportion of female patients was not representative of the proportion of women infected with HIV-1 worldwide, which ranges from $20 \%$ in Europe to $60 \%$ in sub-Saharan Africa. The proportions of patients coinfected with hepatitis $\mathrm{B}$ virus and hepatitis $\mathrm{C}$ virus were $6 \%$ and $7 \%$, respectively.

The primary study endpoint compared the number of patients having an undetectable viral load with a cutoff of $<50$ copies/mL at week 48 . The results were reported at the Conference on Retroviruses and Opportunistic Infections in 2012 by Sax et $\mathrm{al}^{48}$ and published by Wohl et al. ${ }^{49}$ Meanwhile, the data to 96 weeks have been reported and shown stable results. ${ }^{50}$

The drug-related adverse event rate revealed no differences between the study arms with regard to headache, depression and fatigue, upper respiratory infections, and diarrhea. In contrast, abnormal dreams, insomnia, and dizziness together with rash had a much higher incidence during the intake of efavirenz, whereas nausea was reported more often in the Stribild arm. Comparable proportions of patients ( $3 \%$ versus $4 \%$ ) stopped taking their study medication. In the efavirenz arm, $3 \%$ of patients stopped their study medication, mainly due to CNS-related adverse events, and 4\% stopped taking Stribild, mainly (1.4\% versus $0 \%$ ) due to alterations in renal function. ${ }^{49}$

However, the grade 3-4 laboratory adverse event rate, especially elevation of serum creatine kinase, was higher with efavirenz (11\%) than with Stribild (5\%). The remaining grade 3-4 adverse events did not reveal marked differences between the study groups.

Although data on glomerular filtration were not shown in this poster presentation (DeJesus et al, Seattle, WA, USA), ${ }^{51}$ one graph again showed a median serum creatinine increase by $0.14 \mathrm{mg} / \mathrm{dL}$ through week 48 on treatment with Stribild, which is in accordance with previous results from the Phase II studies. ${ }^{16}$

\section{GS- 103}

GS-103, a controlled Phase III clinical trial compared the safety and efficacy of atazanavir boosted with cobicistat $(\mathrm{n}=353)$ versus ritonavir $(\mathrm{n}=355)$ in HIV-1-infected patients, both receiving emtricitabine/tenofovir-DF as a nucleotide backbone, over 48 weeks on treatment. At baseline, patients in both study arms had a comparable mean age (38 versus 39 years), mean baseline HIV viral load (4.88 $\log _{10}$ versus $4.86 \log _{10}$ copies $/ \mathrm{mL}$ ), and median absolute CD4 cell count (364 versus 375 cells $/ \mathrm{mm}^{3}$ ). At baseline, the majority of patients were male ( $92 \%$ and $89 \%$ ) with asymptomatic HIV-1 infection ( $81 \%$ and $83 \%$ ), again under-representing the higher rate of $\mathrm{HIV}$-infected women worldwide. ${ }^{52}$ The proportions of patients coinfected with hepatitis B virus or hepatitis $C$ virus were $5 \%$ and $6 \%$, respectively.

Again, the primary study endpoint compared the number of patients having an undetectable viral load with a cutoff of $<50$ copies $/ \mathrm{mL}$ at week 48 . The results of this study have been published and show comparable results up to week 144. ${ }^{16,48,51,55,-57}$

The drug-related adverse event rate revealed no differences between the study arms with regard to headache, fatigue, upper respiratory infections, diarrhea, and nausea. Interestingly, the ocular icterus which is commonly related to boosted atazanavir treatment showed a significantly lower incidence in the cobicistat arm (1\% versus $14 \%)$. In the cobicistat arm, $4 \%$ of all patients stopped taking their study medication, mainly because of gastrointestinal side effects, and $5 \%$ discontinued in the ritonavir arm, mainly because of icterus, jaundice, or dizziness. 
The proportion of all grade 3-4 laboratory adverse events (in particular elevations in creatine kinase, AST, ALT, and amylase) were comparable between the study arms, whereas hematuria (4\% versus $2 \%)$ and hyperbilirubinemia ( $1 \%$ versus $58 \%, P<0.001)$ were different between the treatment groups.

With regard to kidney function, the authors showed nearly similar median serum creatinine increases of 0.12 versus $0.08 \mathrm{mg} / \mathrm{dL}$ for both groups through the 48 -week treatment period. Only $0.3 \%$ of patients in each study arm stopped treatment early due to renal adverse events.

Although the mean changes in cholesterol revealed statistically significant differences between the two study arms $(P<0.001)$, only $2 \%-4 \%$ of patients in each study group revealed a cholesterol change of grade 2 or more. Despite the differences in total cholesterol, the high/low-density lipoprotein ratio remained roughly the same through week 144, which puts the clinical relevance of such changes into a restricted perspective. $^{53}$

Interestingly, results from a dual-energy X-ray absorptiometry analysis performed in a subgroup of 120 patients at weeks 24 and 48 showed no statistically significant differences between cobicistat-containing and ritonavir-containing treatment: however, a loss of bone mineral density of $2.45 \%$ versus $3.46 \%(P=0.25)$ at the spine and $2.87 \%$ versus $3.59 \%$ $(P=0.12)$ at the hip, with fracture numbers of three versus six $(P=0.51)$ in the study arms at week 48 are not negligible, and most probably related to tenofovir-DF and not to cobicistat, ritonavir, or atazanavir.

\section{Drug-related side effects}

A comparison of the drug-related adverse event rates in the two Phase II studies of Stribild versus Atripla and cobicistat versus ritonavir did not indicate marked differences in the proportions of all grade 1-4 adverse events (Tables 3 and 4). CNS symptoms were not related to either of the pharmacoenhancers, but to efavirenz. The incidence of diarrhea was expectedly highest in patients taking atazanavir/ ritonavir. Again, only marginal differences were detected in both Phase III pivotal trials for all grade 3-4 adverse events, except for the expected much higher rate of hyperbilirubinemia and ocular icterus in the atazanavir-containing arm of GS-103 and CNS-related adverse events with Atripla when compared with Stribild in the GS-102 study. Decreases in bone mineral density were most probably due to taking tenofovir-DF as part of the antiretroviral backbone in Phase III studies.

\section{Treatment-related laboratory abnormalities}

The summary of drug-related grade 2-4 laboratory adverse events are reported if those occurred in more than $5 \%$ of patients or in another sense revealed distinct differences between either Stribild or Atripla and cobicistat-boosted versus ritonavir-boosted therapy regimens (Tables 5 and 6). The cobicistat-related estimated GFR $_{\mathrm{CG}}$ reductions must be appraised, taking into account inhibition of the transmembrane transport pathway of creatinine excretion from blood through the tubular cells into the urinary tract by SLC47A1. Accordingly, it cannot be concluded that the changes in estimated GFR of the first cobicistat presentations shown in $2010^{56}$ proved that GFR decreases in reality. In particular, the findings of a subsequent study of the distinct renal effects of cobicistat that was conducted parallel to the Phase II pivotal trials could explain the mechanisms causing the increased serum creatinine in patients, but also confirm that

Table 3 Summary of adverse events under treatment with combination antiretroviral therapy containing cobicistat from different Phase II studies ${ }^{16,56}$

\begin{tabular}{lllll}
\hline & $\begin{array}{l}\text { EVG/COBI/FTC/TVD } \\
\mathbf{n = 4 8}\end{array}$ & $\begin{array}{l}\text { EFV/FTC/TDF } \\
\mathbf{n = 2 3}\end{array}$ & $\begin{array}{l}\text { COBI/ATV/FTC/TDF } \\
(\mathbf{n = 5 0})\end{array}$ & $\begin{array}{l}\text { RTV/ATV/FTC/TDF } \\
(\mathbf{n = 2 9})\end{array}$ \\
\hline AEs related to randomized drug Grades I-4 & $\mathbf{1 7}(35 \%)$ & $13(57 \%)$ & $10(20 \%)$ & $7(24 \%)$ \\
Grade 3-4 AEs & 0 & $2(9 \%)$ & $2(4 \%)$ & 0 \\
AEs leading to EOS & 0 & $1(4 \%)$ & $2(4 \%)$ & $1(3 \%)$ \\
SAEs & $1(2 \%)$ & $1(4 \%)$ & 0 & $1(3 \%)$ \\
Abnormal dreams, nightmares & $5(10 \%)$ & $8(35 \%)$ & 0 & 0 \\
Dizziness & 0 & $3(13 \%)$ & 0 & 0 \\
Fatigue & $4(8 \%)$ & $3(13 \%)$ & $1(2 \%)$ & $2(7 \%)$ \\
Somnolence & $2(4 \%)$ & $2(9 \%)$ & 0 & 0 \\
Headache & $2(4 \%)$ & $2(9 \%)$ & $1(2 \%)$ & 0 \\
Diarrhea & $4(8 \%)$ & $1(4 \%)$ & $3(6 \%)$ & $3(10 \%)$ \\
Nausea & $2(4 \%)$ & $1(4 \%)$ & $5(10 \%)$ & $1(3 \%)$ \\
\hline
\end{tabular}

Abbreviations: AEs, adverse events; SAEs, serious adverse events; COBI, cobicistat; EVG, elvitegravir; FTC, emtricitabine; TVD, truvada; EFV, efavirenz; TDF, tenofovirdisoproxylfumarate; ATV, atazanavir; RTV ritonavir; EOS, end of study treatment. 
Table 4 Summary of adverse events under treatment with combination antiretroviral therapy containing cobicistat from different Phase III studies

\begin{tabular}{|c|c|c|c|c|}
\hline & $\begin{array}{l}\text { EVG/COBI/FTC/TDF } \\
n=348\end{array}$ & $\begin{array}{l}\text { EFV/FTC/TDF } \\
\mathrm{n}=352\end{array}$ & $\begin{array}{l}\text { COBI/ATV/FTC/TDF } \\
n=353\end{array}$ & $\begin{array}{l}\text { RTV/ATV/FTC/TDF } \\
\mathrm{n}=355\end{array}$ \\
\hline AEs leading to EOS & $4 \%$ & $5 \%$ & $4 \%$ & $5 \%$ \\
\hline Upper respiratory infection & $14 \%$ & $11 \%$ & $15 \%$ & $16 \%$ \\
\hline Rash & $6 \%^{\ddagger}$ & $12 \%^{\ddagger}$ & ND & ND \\
\hline Abnormal dreams & $15 \%$ & $27 \%$ & ND & ND \\
\hline Dizziness & $7 \%^{\S}$ & $24 \% \S$ & ND & ND \\
\hline Fatigue & $12 \%$ & $13 \%$ & $14 \%$ & $13 \%$ \\
\hline Insomnia & $9 \% *$ & $14 \% *$ & ND & ND \\
\hline Depression & $9 \%$ & $12 \%$ & ND & ND \\
\hline Diarrhea & $23 \%$ & $19 \%$ & $22 \%$ & $27 \%$ \\
\hline Nausea & $21 \% *$ & $14 \% *$ & $20 \%$ & $19 \%$ \\
\hline Ocular icterus & ND & ND & $1 \%$ & $14 \%$ \\
\hline Headache & ND & ND & $15 \%$ & $12 \%$ \\
\hline
\end{tabular}

Notes: ${ }^{\ddagger} P=0.009 ; \stackrel{\uparrow}{ } P<0.001 ; * P<0.05$.

Abbreviations: AEs, adverse events; COBI, cobicistat; EVG, elvitegravir; FTC, emtricitabine; EFV, efavirenz; TDF, tenofovir disoproxil fumarate; ATV, atazanavir; RTV ritonavir; EOS, end of study treatment; ND, no data shown.

cobicistat does not influence the real GFR in humans over a longer period of time. ${ }^{57}$

The high rate of increased serum bilirubin, followed by jaundice and ocular icterus seen with the cobicistat-boosted and ritonavir-boosted atazanavir regimens were most probably caused by inhibition of metabolism of bilirubin via UGT1A1 by boosted atazanavir, but not by one of the boosters themselves. Differences in the increase of serum bilirubin were not detected on comparison of the safety results in both study arms, which means that a direct impact of either pharmacoenhancer on serum bilirubin concentrations is not likely and can be verified by previous publications on atazanavir which show a genetic but no atazanavir dose/ exposure-dependent influence on UGT-mediated bilirubin metabolism. $^{58}$

\section{STRATEGY studies}

The two STRATEGY trials were published recently and showed that patients who are on stable NNRTI-based or PIbased cART with a viral load $<50$ copies $/ \mathrm{mL}$ for more than 6 months can switch to Stribild maintenance therapy.

In the randomized, open-label, Phase IIIb STRATEGYNNRTI study, ${ }^{59} 290$ patients switched from either efavirenzbased (83\%) or nevirapine-based cART (17\%) to elvitegravir/ cobicistat/tenofovir/emtricitabine, whereas 143 patients kept taking their former regimen; at week $48,93 \%$ and $88 \%$, respectively, maintained plasma viral loads $<50$ copies $/ \mathrm{mL}$ $(P=0.066)$. There was no treatment-emergent resistance in either group and safety events leading to discontinuation were uncommon. The safety results were comparable with those of the GS-102 study.

Table 5 Summary of grade 2-4 treatment-related laboratory abnormalities occurring in more than $5 \%$ of any treatment arm from different Phase II cobicistat studies ${ }^{56}$

\begin{tabular}{|c|c|c|c|c|}
\hline & $\begin{array}{l}\text { EVG/COBI/FTC/TDF } \\
n=48\end{array}$ & $\begin{array}{l}\text { EFV/FTC/TDF } \\
n=23\end{array}$ & $\begin{array}{l}\text { COBI/ATV/FTC/TDF } \\
(n=50)\end{array}$ & $\begin{array}{l}\text { RTV/ATV/FTC/TDF } \\
(n=29)\end{array}$ \\
\hline Total bilirubin & 0 & 0 & 40 (89\%) & $25(86 \%)^{\S}$ \\
\hline Amylase & $2(4 \%)$ & $2(10 \%)$ & $6(12 \%)$ & $2(7 \%)$ \\
\hline Decreased neutrophils & $3(7 \%)$ & $2(10 \%)$ & I (2\%) & I (3\%) \\
\hline Total cholesterol & $4(9 \%)$ & $2(10 \%)$ & $3(6 \%)$ & 0 \\
\hline Proteinuria & $\mathrm{I}(2 \%)$ & $2(10 \%)$ & $2(4 \%)$ & 0 \\
\hline ALT elevation & 0 & 0 & I (2\%) & I (3\%) \\
\hline AST elevation & 0 & 0 & 0 & I (3\%) \\
\hline Hypophosphatemia & 0 & 0 & I (2\%) & I (3\%) \\
\hline Creatinine elevation & $\mathrm{I}(2 \%)$ & 0 & $6(12 \%)$ & 0 \\
\hline Mean serum creatinine* & $+0.14 \mathrm{mg} / \mathrm{dL}$ & $+0.04 \mathrm{mg} / \mathrm{dL}$ & $+0.18 \mathrm{mg} / \mathrm{dL}$ & $+0.14 \mathrm{mg} / \mathrm{dL}$ \\
\hline Mean eGFR* & $-18 \mathrm{~mL} / \mathrm{min}$ & $-7 \mathrm{~mL} / \mathrm{min}$ & $-15 \mathrm{~mL} / \mathrm{min}$ & $-14 \mathrm{~mL} / \mathrm{min}$ \\
\hline Mean eGFR at week 24 & $111 \mathrm{~mL} / \mathrm{min}$ & $126 \mathrm{~mL} / \mathrm{min}$ & $102 \mathrm{~mL} / \mathrm{min}$ & $111 \mathrm{~mL} / \mathrm{min}$ \\
\hline
\end{tabular}

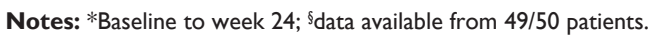

Abbreviations: AST, aspartate transaminase; ALT, alanine transaminase; EFV, efavirenz; ATV, atazanavir; RTV ritonavir; EVG, elvitegravir; COBI, cobicistat; FTC, emtricitabine; TDF, tenofovir-disoproxylfumarate; eGFR, estimated glomerular filtration rate. 
Table 6 Summary of grade 2-4 treatment-related laboratory abnormalities occurring in more than $1 \%$ of any treatment arm from different Phase III cobicistat studies

\begin{tabular}{|c|c|c|c|c|}
\hline & $\begin{array}{l}\text { EVG/COBI/FTC/TDF } \\
n=348\end{array}$ & $\begin{array}{l}\text { EFV/FTC/TDF } \\
\mathrm{n}=352\end{array}$ & $\begin{array}{l}\text { COBI/ATV/FTC/TDF } \\
n=353\end{array}$ & $\begin{array}{l}\text { RTVIATV/FTC/TDF } \\
n=355\end{array}$ \\
\hline Decreased neutrophils & $2 \%$ & $3 \%$ & ND & ND \\
\hline Hematuria & $2 \%$ & $1 \%$ & $4 \%$ & $2 \%$ \\
\hline GGT elevation & $2 \%$ & $5 \%$ & ND & ND \\
\hline ALT elevation & $1 \%$ & $3 \%$ & $2 \%$ & $2 \%$ \\
\hline AST elevation & $2 \%$ & $3 \%$ & $2 \%$ & $3 \%$ \\
\hline Amylase elevation & $2 \%$ & $2 \%$ & $2 \%$ & $3 \%$ \\
\hline Hyperbilirubinemia & ND & ND & $1 \%$ & $58 \%$ \\
\hline Creatine kinase elevation & $5 \%$ & $11 \%$ & $6 \%$ & $7 \%$ \\
\hline Median serum creatinine ${ }^{\S}$ & $+0.14 \mathrm{mg} / \mathrm{dL}$ & $+0.01 \mathrm{mg} / \mathrm{dL}$ & $+0.12 \mathrm{mg} / \mathrm{dL}$ & $+0.08 \mathrm{mg} / \mathrm{dL}$ \\
\hline
\end{tabular}

Note: ${ }^{\circledR}$ Changes in serum creatinine are shown for a period of 48 weeks.

Abbreviations: AST, aspartate transaminase; ALT, alanine transaminase; GGT, gamma-glutamyl transferase; EFV, efavirenz; ATV, atazanavir; RTV ritonavir; EVG, elvitegravir; COBI, cobicistat; FTC, emtricitabine; TDF, tenofovir-disoproxylfumarate; ND, no data shown.

In the randomized, open-label Phase IIIb STRATEGY-PI study, patients who were on stable cART with either darunavir-based or atazanavir-based cART ( $40 \%$ each) switched to Stribild (elvitegravir/cobicistat/tenofovir/emtricitabine), whereas $20 \%$ of patients kept taking their former regimen. The key eligibility criteria included no history of virological failure, no resistance to emtricitabine or tenofovir, and creatinine clearance $\geq 70 \mathrm{~mL} / \mathrm{min}$. After 48 weeks on the new therapy, $93.8 \%$ of 290 patients in the switch groups maintained a viral load $<50$ copies $/ \mathrm{mL}$ versus $87.1 \%$ of 139 in the nonswitch group $(P=0.025)$. The stated statistical superiority of switching was mainly due to the higher proportion of patients in the nonswitch group stopping treatment for nonvirological reasons, such as side effects, eg, icterus with atazanavir/ritonavir or gastrointestinal effects with darunavir/ritonavir. ${ }^{60}$

\section{Renal and hepatic impairment}

Data on the use of cobicistat in patients with hepatic impairment have also been published and indicate no clinically relevant changes in the pharmacokinetics of cobicistat-boosted elvitegravir in patients with mild to moderate hepatic impairment comparable with Child-Pugh status B. While the elvitegravir exposure was moderately enhanced in comparison with that in patients without hepatic impairment, the geometric mean $(90 \%$ confidence interval $[\mathrm{CI}])$ cobicistat AUC and peak plasma concentration ratios were 99.8 (76.0-131) and 86.1 (65.4-113) for patients with hepatic impairment. Hence, cobicistat can be administered in patients with liver impairment without concerns regarding safety and efficacy. ${ }^{61}$

Custodio et al presented data from two randomized crossover cohorts of 32 and 14 healthy volunteers in Amsterdam 2013, where they could show that in the case of combining $150 \mathrm{mg}$ cobicistat and $300 \mathrm{mg}$ tenofovir $(245 \mathrm{mg}$
tenofovir-DF), the tenofovir mean $(90 \% \mathrm{CI})$ maximum plasma concentration is increased 55\% (34-78) compared to tenofovir mean plasma concentrations without coadministration of cobicistat. The mean $(90 \% \mathrm{CI})$ area under the curve (AUC) and trough concentrations for tenofovir were increased 23\% (16-39) and 25\% (16-36), respectively. The authors discussed how cobicistat inhibits intestinal P-glycoprotein efflux and thus enhances the bioavailability of tenofovir-DF. Although a direct correlation between enhanced tenofovir-plasma exposure and decreased renal function has not been shown as yet, patients taking a combination of tenofovir and cobicistat should at least be monitored closely for renal function. ${ }^{62}$

If cobicistat is taken by patients with mild renal impairment, and renal function deteriorates with GFR falling below $50 \mathrm{~mL} / \mathrm{min}$ or serum phosphate levels decreasing to $0.32 \mathrm{mmol} / \mathrm{L}(1.0 \mathrm{mg} / \mathrm{dL})$, cobicistat should be stopped, according to its labeling. If combined with antiretrovirals that have to be dose-adjusted in the event of renal impairment (eg, darunavir, atazanavir), cobicistat should be stopped when the GFR falls below $70 \mathrm{~mL} / \mathrm{min}$. The same counts for a phosphate concentration falling below $<0.48 \mathrm{mmol} / \mathrm{L}$ $(1.5 \mathrm{mg} / \mathrm{dL})$. If a patient takes cobicistat without such combination partners, clinical routine renal function tests should be performed weekly if the GFR drops to $<70 \mathrm{~mL} / \mathrm{min}$.

Cobicistat as part of Stribild should not be taken by patients who have recently taken or are still taking nephrotoxic substances. $^{63}$

\section{Drug interactions}

The renal effects of cobicistat were discussed earlier in this paper. Regarding inhibition of cytochrome oxidases, cobicistat $150 \mathrm{mg}$ has an effect on hepatic CYP3A isoenzymes comparable with that seen with ritonavir $100 \mathrm{mg}$. 
Cobicistat is a substrate and a potent CYP3A inhibitor, whereas its inhibition of CYP2D6 is restricted. It also inhibits cellular transporters such as ABCB1, BCRP, OATP1B1, and OATP1B3, which are located in various blood-tissue barriers and are part of the renal tubular excretion pathways of several drugs. As a consequence, cobicistat could enhance the plasma concentrations of a number of drugs used widely in clinical settings, such as calcium channel blockers, beta-blockers, HMG-CoA reductase inhibitors (statins), antiarrhythmics, sedative hypnotics, so-called lifestyle drugs used for erectile dysfunction and alopecia, corticosteroids, norgestimate, and antiretrovirals. Cobicistat decreases the steady-state clearance of the integrase inhibitor elvitegravir and of several PIs, eg, atazanavir and darunavir. It is marketed as a pharmacokinetic enhancer of elvitegravir (plus tenofovir-DF/emtricitabine, Stribild), and has been coadministered in a number of studies as a pharmacoenhancer of atazanavir as described above. Being a substrate of CYP3A, cobicistat itself is vulnerable to changes in isoenzyme kinetics if other drugs that are coadministered either increase (eg, azole derivatives and clarithromycin) or decrease (eg, rifabutin, carbamazepine, and phenytoin) its plasma exposure. Cobicistat may even simultaneously increase the plasma exposure of the coadministered drugs, if these are not only inhibitors but also substrates of certain CYPs. A list of all interactions with cobicistat being evaluated prior to approval of Stribild is shown in Table $7 .{ }^{64}$ However, information on many clinically relevant drug interactions is not available as yet and should be obtained, especially if dose adjustments may become necessary in (clinically) unavoidable combinations.

Two recent abstracts regarding cobicistat were presented at the 2014 Conference on Retroviruses and Opportunistic Infections in Boston, MA, USA. One presented the bioequivalence of two pediatric formulations versus the adult tablet. One study arm evaluated the pharmacokinetics of $3 \times 50 \mathrm{mg}$ immediate-release tablets versus the $150 \mathrm{mg}$ adult tablet formulation and showed bioequivalence with regard to peak plasma concentration (geometric mean ratio [GMR] 100, 90\% CI 92.8-108) and AUC (GMR 95, 90\% CI 88.9-101), and a second arm compared the pharmacokinetics of $7.5 \times 20 \mathrm{mg}$ (tablet divided using a pill cutter) versus the adult $150 \mathrm{mg}$ tablet formulation and again showing bioequivalence within the protocol-defined specifications for peak plasma concentration (GMR 91.9, 90\% CI 86.8-97.4) and Aci $_{\text {ng }}$ (GMR 96.6, 90\% CI 90.0-104), respectively.

The effect of food on the pharmacokinetics of cobicistat has also been evaluated in a study by Shiomi et al who tested the effects of fasting conditions, a standard breakfast, and a protein-rich drink using a three-way crossover design in healthy volunteers. Administration of a single dose showed decreased plasma concentrations of elvitegravir (50\%) and tenofovir $(28 \%)$ when taken under fasting conditions but no differences between the study groups for emtricitabine and cobicistat. $^{65}$

\section{Pharmacodynamics}

Noninferiority was established for all cobicistat-boosted therapy regimens in the preapproval studies when compared with ritonavir-boosted regimens. Given that cobicistat itself has no antiviral effects, it is redundant to discuss the results of the pivotal studies regarding efficacy. Safety is the important parameter to be discussed in the clinical utility of cobicistat.

However, it is of note that the two Phase IIIb switch studies (STRATEGY-PI and STRATEGY-NNRTI) have shown that patients with maintained suppression of viral load of more than 6 months can switch from a PI-based or NNRTI-based cART to once-daily Stribild as maintenance therapy; both studies showed at least noninferiority of the new cobicistatcontaining regimen versus the former cART. ${ }^{59,60}$ Earlier it was shown that a switch from twice-daily raltegravir-based cART to once-daily Stribild showed comparable safety and efficacy for both regimens. ${ }^{66}$

\section{Discussion}

To appraise the advantages and disadvantages of cobicistat as a pharmacoenhancer of cART one needs to discriminate between pharmacokinetic and pharmacodynamic effects, it must be taken into account that long-term data are not yet available and data are scarce regarding special patient groups, ie, children aged $<12$ years, pregnant women, and elderly patients aged over 65 years.

The CYP3A selectivity of cobicistat metabolism can contribute to better management of drug-drug interactions with cART. The enormous potential of drug-drug interactions involving ritonavir, caused by its coincidental and complex effects on various cytochromes, UGT, and P-glycoprotein, will in all likelihood not be seen with cobicistat and make cART pharmacokinetics much more predictable in future.

However, this will also be to the detriment of the pharmacoenhancing properties of cobicistat. Taking into account that ritonavir-mediated inhibition of P-glycoprotein (ABCB1), especially at the blood-brain barrier, has resulted in cerebrospinal fluid (CSF) PI concentrations at least exceeding the minimum inhibitory concentration for wild-type 


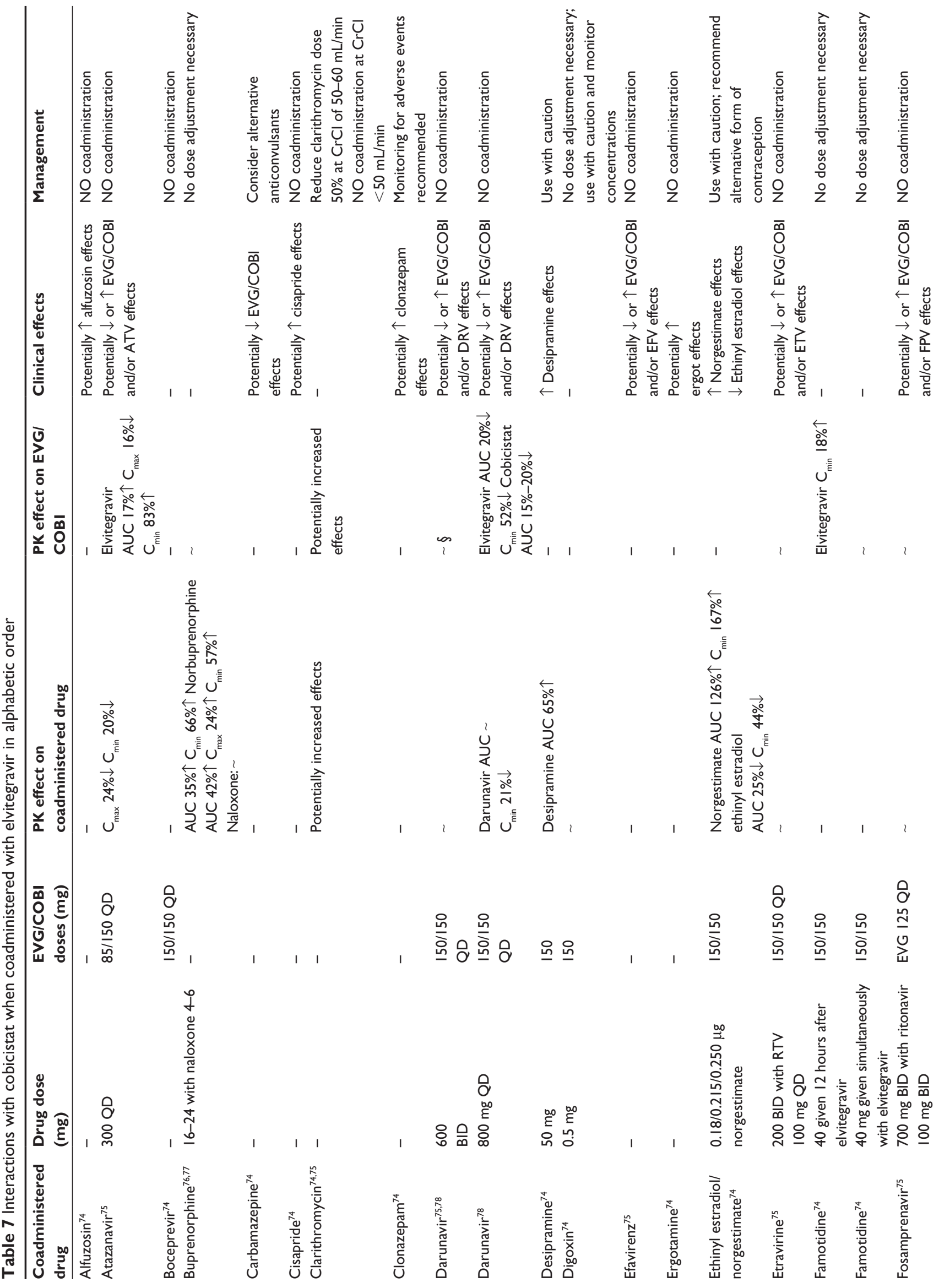



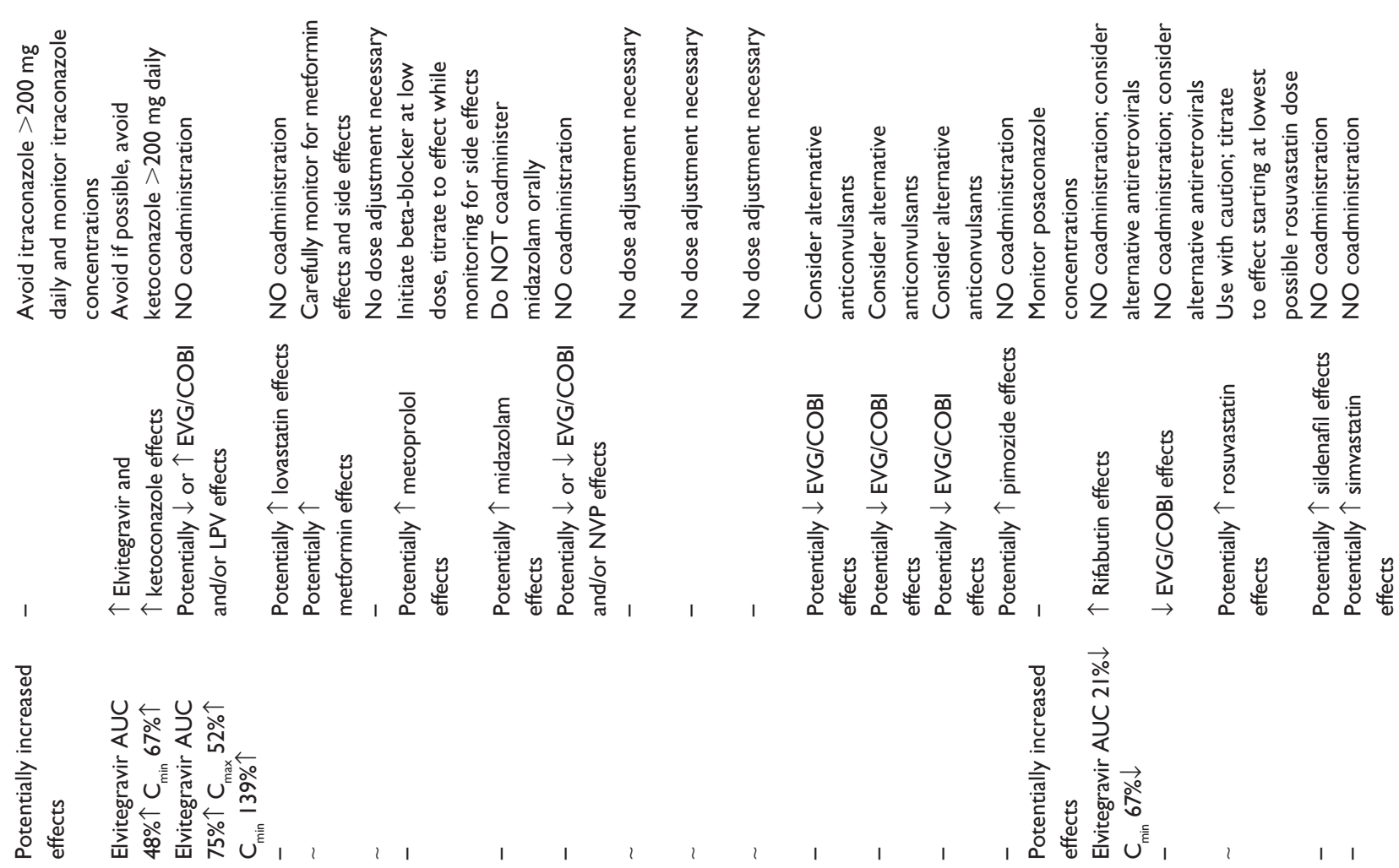

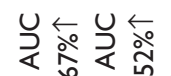

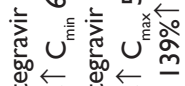

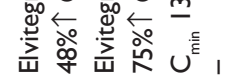

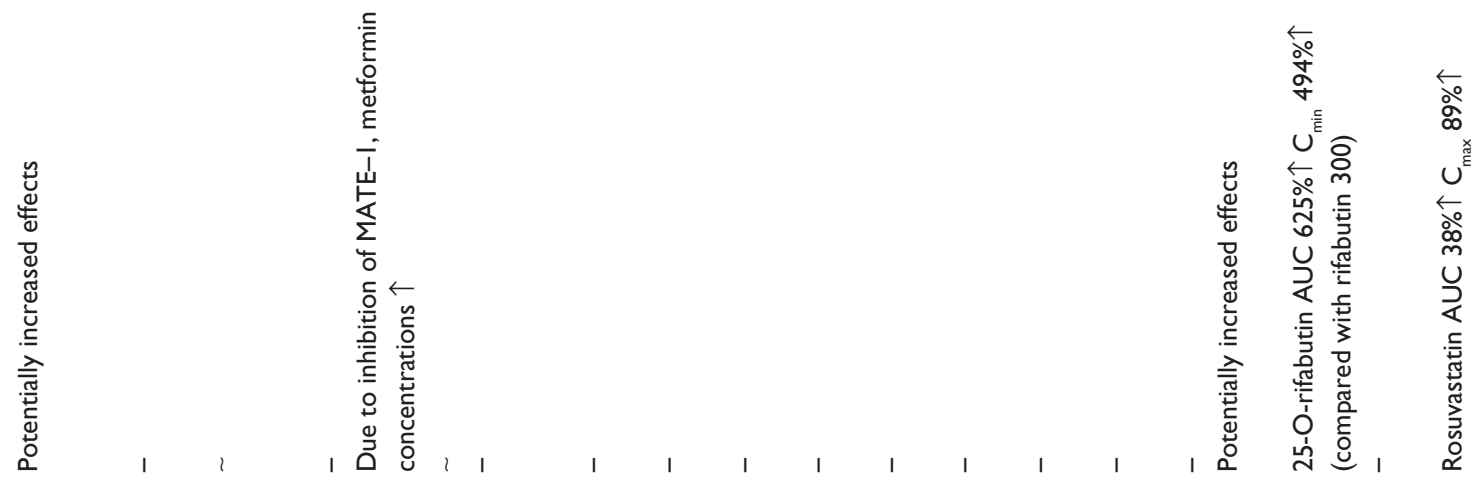

о

용으는

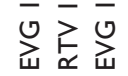

量

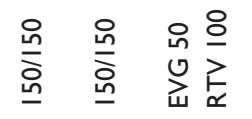

亮, 鿵

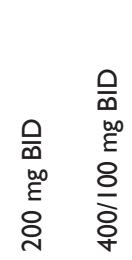

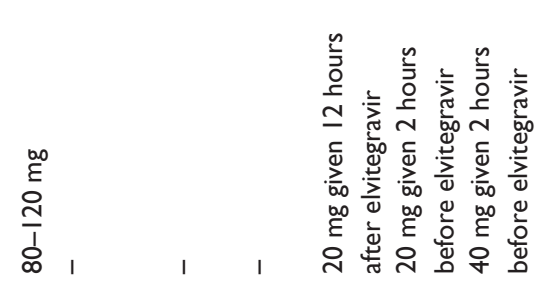

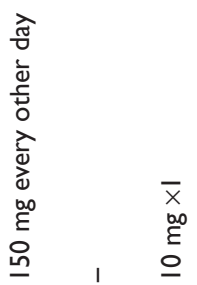

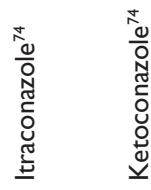
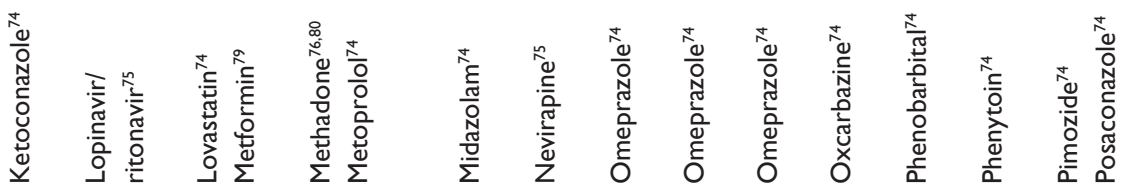

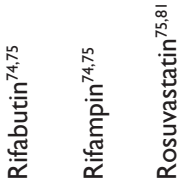

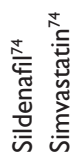




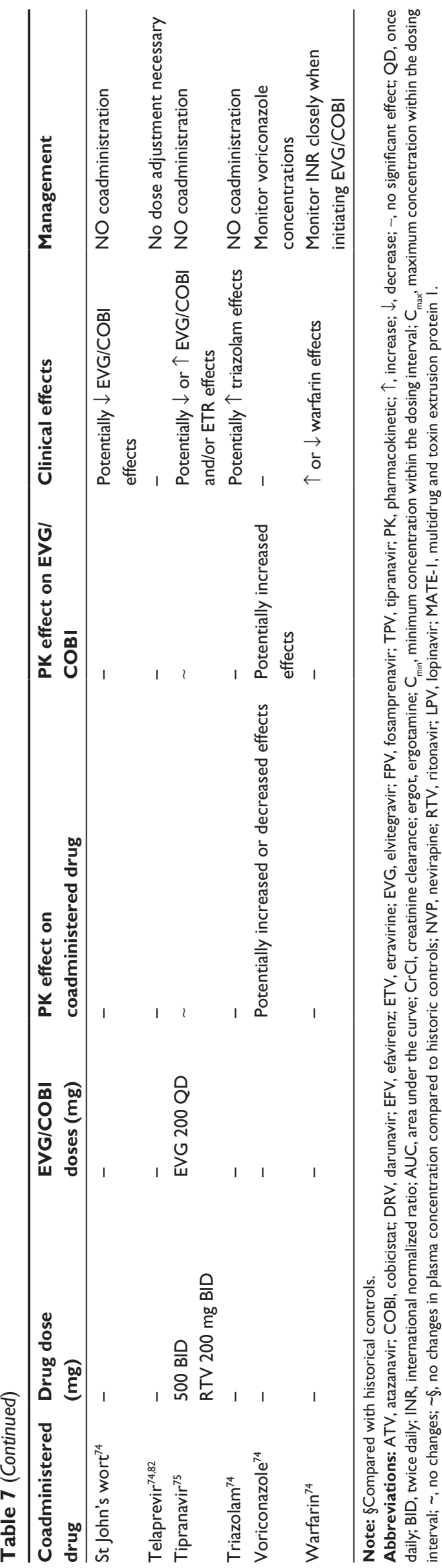

HIV, cobicistat-boosting without P-glycoprotein inhibition may lead to suboptimal PI concentrations in the CSF. For instance, $\mathrm{CSF}$ concentrations of ritonavir-boosted darunavir ${ }^{67}$ and atazanavir ${ }^{68}$ exceed the $\mathrm{IC}_{50}$ of wild-type HIV only by $20 \%-100 \%$, and the effect of P-glycoprotein inhibition has not yet been quantified in this metabolic setting. So far, it is not known whether cobicistat is as effective as ritonavir in inhibiting P-glycoprotein-mediated drug efflux at the bloodbrain barrier. ${ }^{69}$ Currently one study of Scott Letendre is about to start in the U.S.A (www.fda.gov, clinical trials Identifier No. NCT02251236) in order to clarify this question..$^{70}$ There is certainly a strong demand for pharmacokinetic studies addressing this issue.

One handicap of pharmacoenhancement with ritonavir is the high rate of gastrointestinal side effects, especially chronic diarrhea if combined with PIs which cause low plasma ritonavir concentrations and probably high gut concentrations of not resorbed ritonavir, as shown with lopinavir, amprenavir, and saquinavir. ${ }^{71}$ HIV PI combinations accounting for high ritonavir plasma concentrations, ie, atazanavir/ritonavir or darunavir/ ritonavir, have a much lower prevalence of gastrointestinal side effects. However, the counterpart of ritonavir and, cobicistat, may also cause gastrointestinal side effects such as nausea and vomiting, but causes diarrhea to a much lower extent and most probably due to the pharmacological properties of the antiretrovirally effective combination partners. In the long run, this could considerably increase the quality of life for many patients suffering from chronic diarrhea caused by cART over years on treatment.

In the early Phase II studies, cobicistat revealed an increase of serum creatinine with a subsequently calculated dose-dependent and time-dependent decrease in GFR in study patients. ${ }^{16,72}$ The focus was put on a possible negative effect on renal function in patients. The detected serum creatinine increases, which reached a plateau after 4 weeks on therapy and remained unchanged until week 48 in the early pivotal trials, affected the Cockcroft-Gault formulabased calculation of estimated GFR, which is used in most clinical settings for kidney diagnostics. However, it could be demonstrated that the real $\mathrm{GFR}^{73}$ was not influenced at all, as shown by stable iohexol clearance. The mechanism behind this effect is a cobicistat concentration-dependent inhibition of the renal transmembrane transporter SLC47A1, which is located at the apical tubular cell membrane and is part of the excretion pathway of creatinine. Indeed, this phenomenon has been already described for other drugs such as cimetidine, trimethoprim, and dolutegravir. A following investigation of the impact on proximal renal tubular 


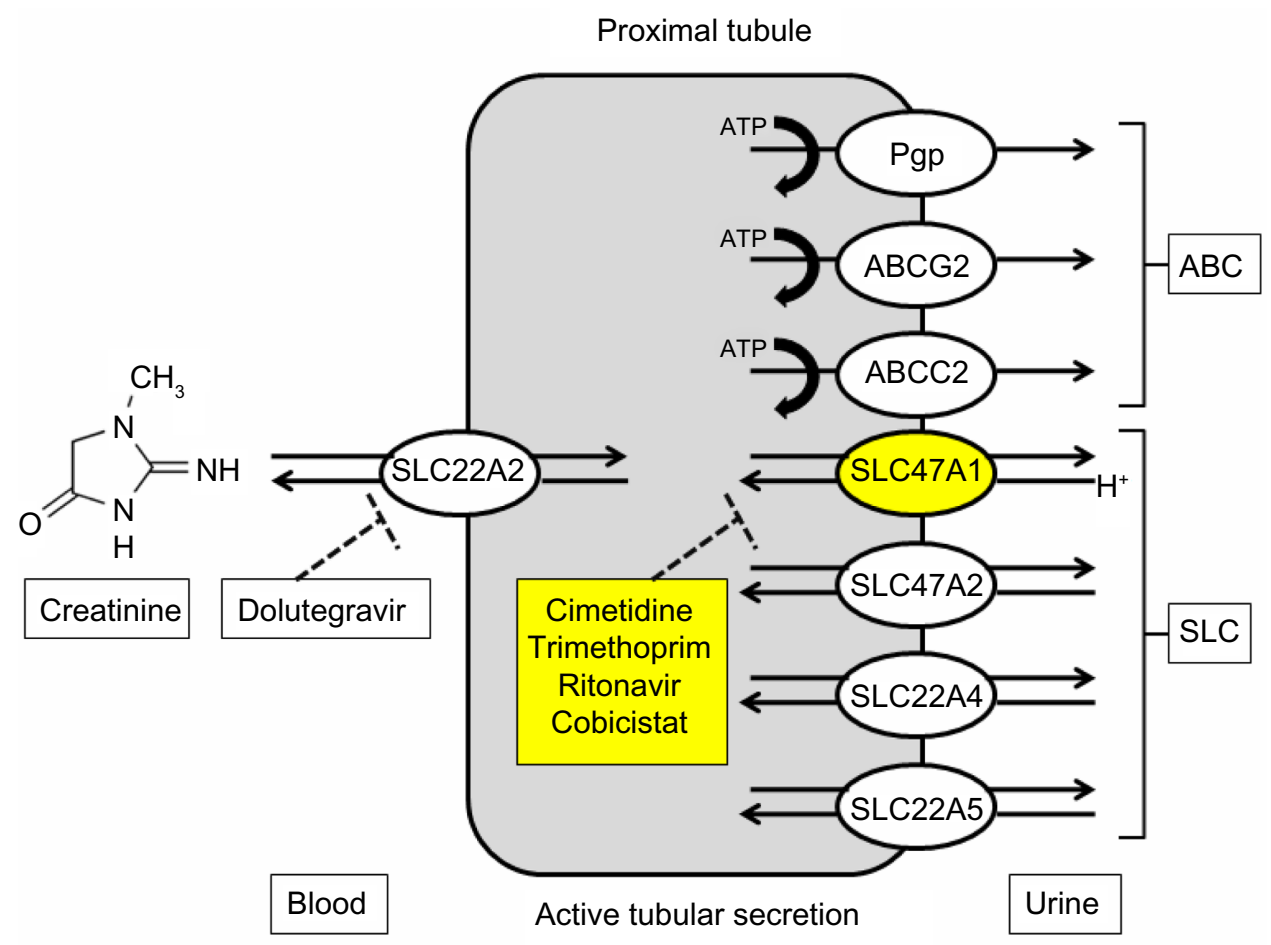

Figure I Pathway of renal creatinine transport via efflux transporters SLC22A2 and SLC47AI over the proximal tubular cell membranes from blood to urine. Abbreviations: ABC, ATP-binding cassette; SLC, solute carrier; PgP, P-glycoprotein; ABCG2, breast cancer resistance protein; ABCC2, multidrug resistance protein; SLC47AI, multidrug and toxin extrusion protein I; SLC47A2, multidrug and toxin extrusion protein 2; SLC22A4, organic cation/ergothioneine transporter I; SLC22A5, organic cation/ergothioneine transporter 2; SLC22A2, organic cation transporter 2.

transporters could show that cobicistat, ritonavir, cimetidine, and trimethoprim inhibit the SLC47A1 transporter at comparable $\mathrm{IC}_{50}$ values of $1.87,1.34,0.95$, and $3.31 \mu \mathrm{M}$ (Figure 1). However, this does not explain differences between cobicistat and ritonavir as described before. One possible explanation is the different concentrations reached by cobicistat or ritonavir at the proximal tubular membrane compartment. ${ }^{56}$ The clinical relevance of this phenomenon is not direct but indirect, so clinicians must discriminate between cobicistat-related creatinine elevations and real tubular damage. Creatinine is no more a leading parameter for that and further routine controls must be performed, eg, urinary protein and serum phosphate.

After so many years, a strong competitor of ritonavir has entered the stage. Cobicistat is a long expected alternative pharmacoenhancer for cART probably leading to easier handling of combination pharmacotherapy and predictability of drug-drug interactions with cART in many conceivable drug combinations. Cobicistat has not yet revealed a high rate of undesirable or unexpected side effects. However, not having completed its second year on the market, the utility of cobicistat as pharmacoenhancer of cART has to be appraised with caution; in comparison with ritonavir, a much smaller number of patients have been exposed to cobicistat for a comparably short time to date. In particular, the proportion of female volunteers and patients in the preapproval trials does not correspond with the proportion of women infected with HIV-1 worldwide, ${ }^{50}$ so more studies are required on the long-term safety in women, especially those of childbearing age.

Data on pregnant women, younger children, and patients aged over 65 years are scarce. Although categorized as "B" in the US Food and Drug Administration pregnancy categories due to the drugs preclinical safety profile, real life data during pregnancy as well as studies in special patient groups are in demand to gain more safety in prescription and handling situations, like the inception of pregnancy while being on a cobicistat-containing cART. Cobicistat has been evaluated and was approved as a pharmacoenhancer in only one fixed-drug combination as yet, but is already under evaluation for accessory fixed-dose combinations. However, its timely availability as a single tablet that could be individually combined with other antiretrovirals would be desirable.

\section{Disclosure}

The author has received fees for educational lectures from AbbVie, Jannssen Cilag, ViiV Healthcare and GILEAD 
through the past five years. The author has not received travel expensions or honorariums for any kind of scientific work. The author declares no conflicts of interest in this work.

\section{References}

1. Lee L, Karon J, Selik R, Neal J, Fleming P. Survival after AIDS diagnosis in adolescents and adults during the treatment era, United States, 1984-1997. JAMA. 1998;285:1308-1315.

2. Mocroft A, Ledergerber B, Katlama C, et al. Decline in the AIDS and death rates in the EuroSIDA study: an observational study. Lancet. 2003;362:22-29.

3. Collot-Teixeira S, De Lorenzo F, Waters L, et al. Impact of different lowdose ritonavir regimens on lipids, CD36, and adipophilin expression. Clin Pharmacol Ther. 2009;85:375-378.

4. Carr A. Cardiovascular risk factors in HIV-infected patients. J Acquir Immune Defic Syndr. 2003;34 Suppl 1:S73-S78.

5. Sax PE, Kumar P. Tolerability and safety of HIV protease inhibitors in adults. J Acquir Immune Defic Syndr. 2004,37:1111-1124.

6. Friis-Moller N, Reiss P, Sabin CA, et al. Class of antiretroviral drugs and the risk of myocardial infarction. $N$ Engl J Med. 2007;356: 1723-1735.

7. Morgello S, Mahboob R, Yakoushina T, Khan S, Hague K. Autopsy findings in a human immunodeficiency virus-infected population over 2 decades: influences of gender, ethnicity, risk factors, and time. Arch Pathol Lab Med. 2002;126:182-190.

8. Friis-Moller N, Sabin C, Weber R, et al. Combination antiretroviral therapy and the risk of myocardial infarction. N Engl J Med. 2003;349: 1993-2003.

9. Perronne C. Antiviral hepatitis and antiretroviral drug interactions. J Hepatol. 2006;44:S119-S125.

10. Wilby KJ, Greanya ED, Ford JA, Yoshida EM, Partovi N. A review of drug interactions with boceprevir and telaprevir: implications for HIV and transplant patients. Ann Hepatol. 2012;11:179-185.

11. Vachon ML, Dieterich DT. The HIV/HCV-coinfected patient and new treatment options. Clin Liver Dis. 2011;15:585-596.

12. Soriano V, Sherman KE, Rockstroh J, et al. Challenges and opportunities for hepatitis C drug development in HIV-hepatitis C virus-co-infected patients. AIDS. 2011;25:2197-2208.

13. Seden K, Back D, Khoo S. New directly acting antivirals for hepatitis C: potential for interaction with antiretrovirals. J Antimicrob Chemother. 2010;65:1079-1085.

14. Hsu A, Granneman GR, Bertz RJ. Ritonavir. Clinical pharmacokinetics and interactions with other anti-HIV agents. Clin Pharmacokinet. 1998;35:275-291.

15. Gallant JE. Protease-inhibitor boosting in the treatment-experienced patient. AIDS Rev. 2004;6:226-233.

16. Cohen C, Elion R, Ruane P, et al. Randomized, phase 2 evaluation of two single-tablet regimens elvitegravir/cobicistat/emtricitabine/ tenofovir disoproxil fumarate versus efavirenz/emtricitabine/tenofovir disoproxil fumarate for the initial treatment of HIV infection. AIDS. 2011;25:F7-F12.

17. Elion R, Cohen C, Gathe J, et al. Phase 2 study of cobicistat versus ritonavir each with once-daily atazanavir and fixed-dose emtricitabine/tenofovir df in the initial treatment of HIV infection. AIDS. 2011;25:1881-1886.

18. Roberts N, Martin J, Kinchington D, et al. Rational design of peptidebased HIV proteinase inhibitors. Science. 1990;248:358-361.

19. Danner S, Carr A, Leonard J, et al; European-Australian Collaborative Ritonavir Study Group. A short-term study of the safety, pharmacokinetics, and efficacy of ritonavir, an inhibitor of HIV-1 protease. $N$ Engl J Med. 1995;333:1528-1533.

20. Markowitz M, Saag M, Powderly WG, et al. A preliminary study of ritonavir, an inhibitor of HIV-1 protease, to treat HIV-1 infection. N Engl J Med. 1995;333:1534-1539.
21. Lorenzi P, Yerly S, Abderrakim K, et al. Toxicity, efficacy, plasma drug concentrations and protease mutations in patients with advanced HIV infection treated with ritonavir plus saquinavir. Swiss HIV Cohort Study. AIDS. 1997;11:F95-F99.

22. Merry C, Barry MG, Mulcahy F, et al. Saquinavir pharmacokinetics alone and in combination with ritonavir in HIV-infected patients. AIDS. 1997;11:F29-F33.

23. Hoetelmans RM, Meenhorst PL, Mulder JW, Burger DM, Koks CH, Beijnen JH. Clinical pharmacology of HIV protease inhibitors: focus on saquinavir, indinavir, and ritonavir. Pharm World Sci. 1997;19: 159-175.

24. Hsu A, Granneman GR, Cao G, et al. Pharmacokinetic interaction between ritonavir and indinavir in healthy volunteers. Antimicrob Agents Chemother. 1998;42:2784-2791.

25. Buscher A, Hartman C, Kallen MA, Giordano TP. Impact of antiretroviral dosing frequency and pill burden on adherence among newly diagnosed, antiretroviral-naive HIV patients. Int J STD AIDS. 2012;23: 351-355.

26. Juday T, Gupta S, Grimm K, Wagner S, Kim E. Factors associated with complete adherence to HIV combination antiretroviral therapy. HIV Clin Trials. 2011;12:71-78.

27. Kauf TL, Davis KL, Earnshaw SR, Davis EA. Spillover adherence effects of fixed-dose combination HIV therapy. Patient Prefer Adherence. 2012;6:155-164.

28. Sterrantino G, Santoro L, Bartolozzi D, Trotta M, Zaccarelli M. Self-reported adherence supports patient preference for the single tablet regimen (STR) in the current cART era. Patient Prefer Adherence. 2012;6:427-433.

29. Bangalore S, Kamalakkannan G, Parkar S, Messerli FH. Fixed-dose combinations improve medication compliance: a meta-analysis. Am J Med. 2007;120:713-719.

30. German P, Warren D, West S, Hui J, Kearney BP. Pharmacokinetics and bioavailability of an integrase and novel pharmacoenhancer-containing single-tablet fixed-dose combination regimen for the treatment of HIV. J Acquir Immune Defic Syndr. 2010;55:323-329.

31. Ramanathan S, Warren D, Wei L, Hui J, Kearney B. Pharmacokinetic boosting of atazanavir with the pharmacoenhancer GS-9350 versus ritonavir. Presented at the 49th Interscience Conference on Antimicrobial Agents and Chemotherapy, September 12-15, 2009, San Francisco, CA, USA.

32. Gallant JE, Koenig E, Andrade-Villanueva J, et al. Cobicistat versus ritonavir as a pharmacoenhancer of atazanavir plus emtricitabine/tenofovir disoproxil fumarate in treatment-naive HIV type 1-infected patients: week 48 results. J Infect Dis. 2013;208: 32-39.

33. Mathias A, Liu H, Warren DR, Sekar VJ, Kearney BP. Relative bioavailability and pharmacokinetics of darunavir when boosted with the pharmacoenhancer GS-9350 versus ritonavir. Presented at the 11th International Workshop Clinical Pharmacology of HIV Therapy, April 7-9, 2010, Sorrento, Italy.

34. Kakuda T, van de Casteele T, Petrovic R, Osomer M, Tomaka F, Hoetelmans R. Bioequivalence of darunavir/cobicistat fixed-dose combination tablet (FDC) versus single agents in healthy volunteers. Presented at the 14th International Workshop on Clinical Pharmacology, April 22-24, 2013, Amsterdam, The Netherlands.

35. Cooper C, van Heeswijk R, Gallicano K, Cameron D. A review of lowdose ritonavir in protease inhibitor combination therapy. Clin Infect Dis. 2003;36:1585-1592.

36. Eagling V, Back D, Barry M. Differential inhibition of cytochrome P450 isoforms by the protease inhibitors ritonavir, saquinavir and indinavir. Br J Clin Pharmacol. 1997;44:190-194.

37. Hsu A, Grannemann G, Bertz R. Ritonavir. Clinical pharmacokinetics and interactions with other anti-HIV agents. Clin Pharmacokinet. 1998;35:275-291.

38. Hsu A, Grannemann G, Witt G, et al. Multiple-dose pharmacokinetics of ritonavir in human immunodefficiency virus-infected subjects. Antimicrob Agents Chemother. 1997;41:898-905. 
39. Granfors M, Wang J, Kajosaari L, Laitila J, Neuvonen P, Backman J. Differential inhibition of cytochrome P450 3A4, 3A5 and 3A7 by five human immunodeficiency virus (HIV) protease inhibitors in vitro. Basic Clin Pharmacol Toxicol. 2006;98:79-85.

40. Kumar G, Dykstra J, Roberts E, et al. Potent inhibition of the cytochrome P-450 3A-mediated human liver microsomal metabolism of a novel HIV protease inhibitor by ritonavir: a positive drug-drug interaction. Drug Metab Dispos. 1999;27:902-908.

41. Kaletra Fachinformation. Abbott Laboratories Ltd. Berkshire, UK. EU/1/01/172/004 EU/1/01/172/005EU/1/01/172/007 2010,20.03.2010. German.

42. von Hentig N, Lotsch J. Cytochrome P450 3A inhibition by atazanavir and ritonavir, but not demography or drug formulation, influences saquinavir population pharmacokinetics in human immunodeficiency virus type 1-infected adults. Antimicrob Agents Chemother. 2009;53: 3524-3527.

43. Crommentuyn K, Kappelhoff B, Mulder J, et al. Population pharmacokinetics of lopinavir in combination with ritonavir in HIV-1infected patients. Br J Clin Pharmacol. 2005;60:378-389.

44. Solas C, Poizot-Martin I, Drogoul M, et al. Therapeutic drug monitoring of lopinavir/ritonavir given alone or with a non-nucleoside reverse transcriptase inhibitor. Br J Clin Pharmacol. 2004;57:436-440.

45. Hsu A, Isaacson J, Brun S, et al. Pharmacokinetic-pharmacodynamic analysis of lopinavir-ritonavir in combination with efavirenz and two nucleoside reverse transcriptase inhibitors in extensively pretreated human immunodeficiency virus-infected patients. Antimicrob Agents Chemother. 2003;47:350-359.

46. German P, Lui C, Warren D, et al. Effect of cobicistat on glomerular filtration rate (GFR) in subjects with normal and impaired renal function. Abstract presented at the 51st Interscience Conference on Antimicrobial Agents and Chemotherapy, September 17-20, 2001, Chicago, IL, USA.

47. Elion R, Gathe J, Rashbaum B. The single-tablet regimen elvitegravir/ cobicistat/emtricitabine/tenofovir disproxil fumarate (EVG/COBI/FTC/ TDF; "QUAD") maintains a high rate of virologic suppression and cobicistat (COBI) is an effective pharmacoenhancer through 48 weeks. Presented at the 50th Interscience Conference on Antimicrobial Agents and Chemotherapy, September 12-15, 2010, Chicago, IL, USA.

48. Sax P, DeJesus E, Mills A, Zolopa A, Cohen C, Wohl D, et al. Elvitegravir/Cobicistat/Emtricitabine/Tenofovir (Quad) Has Non-inferior Efficacy and Favorable Safety Compared to Efavirenz/Emtricitabine/ Tenofovir in Treatment-naïve HIV-1+ Subjects. Conference on Retroviruses and Opportunistic Infections. 2012, Seattle, USA:Abstract 101.

49. Wohl DA, Cohen C, Gallant JE, et al. A randomized, double-blind comparison of single-tablet regimen elvitegravir/cobicistat/emtricitabine/ tenofovir DF versus single-tablet regimen efavirenz/emtricitabine/ tenofovir DF for initial treatment of HIV-1 infection: analysis of week 144 results. J Acquir Immune Defic Syndr. 2014;65:e118-e120.

50. Zolopa A, Sax PE, DeJesus E, et al. A randomized double-blind comparison of coformulated elvitegravir/cobicistat/emtricitabine/tenofovir disoproxil fumarate versus efavirenz/emtricitabine/tenofovir disoproxil fumarate for initial treatment of HIV-1 infection: analysis of week 96 results. J Acquir Immune Defic Syndr. 2014;63:96-100.

51. De Jesus E, Rockstroh J, Henry K, Molina JM, Gathe J, Ramanathan S, et al. Efficacy and safety of Elvitegravir/Cobicistat/Emtricitabine/ tenofovir DF "Quad” Compared to Ritonavir-boosted Atazanavir plus Emtricitabine/Tenofovir DF in Treatment Naive HIV-1 Infected Subjects. Conference on Retroviruses and Opportunistic Infections. 2012, Seattle, USA:Poster 627

52. United Nations Programme on HIV/AIDS. UNAIDS report on the global epidemic of HIV, 2013. Available from: http://www.unaids.org/ en/media/unaids/contentassets/documents/epidemiology/2013/gr2013/ UNAIDS_Global_Report_2013_en.pdf. Accessed October 3, 2014.

53. Clumeck N, Molina JM, Henry K, et al. A randomized, double-blind comparison of single-tablet regimen elvitegravir/cobicistat/emtricitabine/tenofovir DF vs ritonavir-boosted atazanavir plus emtricitabine/ tenofovir DF for initial treatment of HIV-1 infection: analysis of week 144 results. J Acquir Immune Defic Syndr. 2014;65:e121-e124.
54. Rockstroh JK, DeJesus E, Henry K, et al. A randomized, double-blind comparison of coformulated elvitegravir/cobicistat/emtricitabine/tenofovir DF vs ritonavir-boosted atazanavir plus coformulated emtricitabine and tenofovir DF for initial treatment of HIV-1 infection: analysis of week 96 results. J Acquir Immune Defic Syndr. 2014;62:483-486.

55. DeJesus E, Rockstroh JK, Henry K, et al. Co-formulated elvitegravir, cobicistat, emtricitabine, and tenofovir disoproxil fumarate versus ritonavir-boosted atazanavir plus co-formulated emtricitabine and tenofovir disoproxil fumarate for initial treatment of HIV-1 infection: a randomised, double-blind, phase 3, non-inferiority trial. Lancet. 2012;379:2429-2438.

56. Cohen C, Shamblaw D, Ruane P, et al. Single-tablet, fixed-dose regimen of elvitegravir/emtricitabine/tenofovir disoproxil fumarate/GS-9350 achieves a high rate of virologic suppression and GS-9350 is an effective booster. Presented at the 17th Conference on Retroviruses and Opportunistic Infections, February 16-19, 2010, San Francisco, CA, USA.

57. Lepist EI, Zhang X, Hao J, et al. Contribution of the organic anion transporter OAT2 to the renal active tubular secretion of creatinine and mechanism for serum creatinine elevations caused by cobicistat. Kidney Int. 2014;86:350-357.

58. Rodriguez-Novoa S, Martin-Carbonero L, Barreiro P, et al. Genetic factors influencing atazanavir plasma concentrations and the risk of severe hyperbilirubinemia. AIDS. 2007;21:41-46.

59. Pozniak A, Markowitz M, Mills A, et al. Switching to coformulated elvitegravir, cobicistat, emtricitabine, and tenofovir versus continuation of non-nucleoside reverse transcriptase inhibitor with emtricitabine and tenofovir in virologically suppressed adults with HIV (STRATEGYNNRTI): 48 week results of a randomised, open-label, phase $3 \mathrm{~b}$ noninferiority trial. Lancet Infect Dis. 2014;14:590-599.

60. Arribas JR, Pialoux G, Gathe J, et al. Simplification to coformulated elvitegravir, cobicistat, emtricitabine, and tenofovir versus continuation of ritonavir-boosted protease inhibitor with emtricitabine and tenofovir in adults with virologically suppressed HIV (STRATEGY-PI): 48 week results of a randomised, open-label, phase $3 \mathrm{~b}$, non-inferiority trial. Lancet Infect Dis. 2014;14:581-589.

61. Custodio JM, Rhee M, Shen G, Ling KH, Kearney BP, Ramanathan S. Pharmacokinetics and safety of boosted elvitegravir in subjects with hepatic impairment. Antimicrob Agents Chemother. 2014;58: 2564-2569.

62. Custodio J, Garner W, Jin F, et al. Evaluation of the drug interaction potential between the pharmacokinetic enhancer cobicistat and tenofovir disoproxil fumarate in healthy subjects. Presented at the 14th International Workshop on Clinical Pharmacology of HIV Therapy, April 22-24, 2013, Amsterdam, The Netherlands.

63. European Medicines Agency. Zusammenfassung der Merkmale des Arzneimittels Stribild. http://www.ema.europa.eu/docs/de_DE/ document_library/EPAR_-(SPc in German)_Product_Information/ human/002574/WC500144272.pdf. Accessed July 20, 2014.

64. University of California, San Francisco. All interactions with elvitegravir/cobicistat, 2013. Available from: http:/hivinsite.ucsf. edu/insite? page $=$ ar-00-02\&param $=225 \&$ post $=4$. Accessed October 3, 2014.

65. Shiomi M, Matsuki S, Ikeda A, et al. Effects of a protein-rich drink or a standard meal on the pharmacokinetics of elvitegravir, cobicistat, emtricitabine and tenofovir in healthy Japanese male subjects: a randomized, three-way crossover study. J Clin Pharmacol. 2014;54: 640-648.

66. Mills A, Crofoot G, Ortiz R, et al. Switching from twice-daily raltegravir plus tenofovir disoproxil fumarate/emtricitabine to once-daily elvitegravir/cobicistat/emtricitabine/tenofovir disoproxil fumarate in virologically suppressed, HIV-1-infected subjects: 48 weeks data. $H I V$ Clin Trials. 2014;15:51-56.

67. Capparelli EV, Holland D, Okamoto C, et al. Lopinavir concentrations in cerebrospinal fluid exceed the $50 \%$ inhibitory concentration for HIV. AIDS. 2005;19:949-952.

68. Best BM, Letendre SL, Brigid E, et al. Low atazanavir concentrations in cerebrospinal fluid. AIDS. 2009;23:83-87. 
69. Letendre S. Central nervous system complications in HIV disease: HIV-associated neurocognitive disorder. Top Antivir Med. 2012;19: 137-142.

70. Lepist EI, Phan TK, Roy A, et al. Cobicistat boosts the intestinal absorption of transport substrates, including HIV protease inhibitors and GS-7340, in vitro. Antimicrob Agents Chemother. 2012;56: 5409-5413.

71. European Medicines Agency 2009, Dok.-Ref.: EMA/793683/2009 EMEA/H/C/127:Last update 01/2010, Accessed 2025.2003.2012.

72. Mathias AA, German P, Murray BP, et al. Pharmacokinetics and pharmacodynamics of GS-9350: a novel pharmacokinetic enhancer without anti-HIV activity. Clin Pharmacol Ther. 2012;87: 322-329.

73. Mathias AA, German P, Murray BP, et al. Pharmacokinetics and pharmacodynamics of GS-9350: a novel pharmacokinetic enhancer without anti-HIV activity. Clin Pharmacol Ther. 2010;87: 322-329.

74. Stribild Package Insert. Foster City, CA, USA: Gilead Sciences, Inc.; 2012. Available from: http://www.gilead.com/ /media/Files/pdfs/ medicines/hiv/stribild/stribild_pi.pdf. Accessed October 3, 2014.

75. Department of Health and Human Services. Guidelines for the use of antiretroviral agents in pediatric HIV infection, February 12, 2013. Available from: http://www.aidsinfo.nih.gov/contentfiles/pediatricguidelines.pdf. Accessed February 2, 2015.

76. Bruce RD, Winkle P, Custodio J. Pharmacokinetics of cobicistatboosted elvitegravir administered in combination with methadone or buprenorphine/naloxone. Presented at the 52nd Interscience Conference on Antimicrobial Agents and Chemotherapy, September 9-12, 2012, San Francisco, CA, USA.
77. Bruce RD, Winkle P, Custodio JM, et al. The pharmacokinetic and pharmacodynamic interactions between buprenorphine/naloxone and elvitegravir/cobicistat in subjects receiving chronic buprenorphine/ naloxone treatment. J Acquir Immune Defic Syndr. 2014;63: 480-484.

78. Ramanathan S, Wang H, Szwarcberg J, Kearney BP. Safety/tolerability, pharmacokinetics and boosting of twice-daily cobicistat administered alone or in combination with darunavir or tipranavir. Presented at the13th International Workshop on Clinical Pharmacology of HIV Therapy, April 16-18, 2012, Barcelona, Spain.

79. Gilead Sciences Ltd. SPC for Tybost $150 \mathrm{mg}$ film coated tablet. Available from: http://www.medicines.org.uk/emc/medicine/28298. Accessed July 20, 2014.

80. Bruce RD, Winkle P, Custodio JM, et al. Investigation of the interactions between methadone and elvitegravir-cobicistat in subjects receiving chronic methadone maintenance. Antimicrob Agents Chemother. 2014;57:6154-6157.

81. Custodio JM, Wang H, Hao J, et al. Pharmacokinetics of cobicistat boosted-elvitegravir administered in combination with rosuvastatin. J Clin Pharmacol. 2014;54:649-656.

82. Custodio J, Guo S, Lawson E, et al. Lack of clinically relevant drug interactions between elvitegravir/cobicistat/emtricitabine/tenofovir disoproxil fumarate and telaprevir 2013. Poster presented at the 53rd Interscience Conference on Antimicrobial Agents and Chemotherapy, September 10-13, Denver, CO, USA.

83. University of California, San Francisco. All interactions with elvitegravir/cobicistat 2013. Available from: http://hivinsite.ucsf. edu/insite?page $=$ ar $-00-02 \&$ param $=225 \&$ post $=4$. Accessed June 20, 2014 .
HIV/AIDS - Research and Palliative Care

\section{Publish your work in this journal}

HIV/AIDS - Research and Palliative Care is an international, peerreviewed open-access journal focusing on advances in research in HIV, its clinical progression and management options including antiviral treatment, palliative care and public healthcare policies to control viral spread. The journal welcomes original research, basic science,

\section{Dovepress}

clinical \& epidemiological studies, reviews \& evaluations, expert opinion \& commentary, case reports \& extended reports. The manuscript management system is completely online and includes a very quick and fair peer-review system. Visit http://www.dovepress.com/ testimonials.php to read real quotes from published authors. 